\title{
Acompañamiento psicosocial en dos comunidades de Manabí tras el terremoto del 16 de abril: una perspectiva desde la salud colectiva y los derechos humanos
}

Fernanda Soliz,* Antonio J. Canela-Ruano,**

Paula Yépez-Tito, ${ }^{* * *}$ Lorena Pérez,**** Carlos Reyes,*****

\section{RESUMEN}

El artículo presenta dos experiencias de acompañamiento psicosocial que se realizaron en la provincia de Manabí en Ecuador en los meses posteriores al terremoto del 16 de abril de 2016. La primera transcurrió en el barrio Ébano en Jama, y la segunda en el barrio Los Cañonazos en Portoviejo. En ambos casos, el acompañamiento se orientó al fortalecimiento del tejido comunitario que pone de manifiesto los efectos perversos asociados a los desastres, tales como la concentración de tierras o la discriminación ambiental. Para esto, se describen las fases de puesta de contacto, las actividades realizadas, la metodología utilizada y las conclusiones sobre el acompañamiento realizado, bajo una perspectiva de la salud colectiva y derechos humanos.

Palabras Clave: terremoto Ecuador, Manabí, acompañamiento psicosocial, salud colectiva, derechos humanos.

\section{ABSTRACT}

This paper intends to present two experiences of psychosocial accompaniment that were carried out in the province of Manabí, Ecuador in the next months following the earthquake of April 16, 2016. The first one takes place in the neighborhood Ebony in Jama and the second in the neighborhood Los Cañonazos in

\footnotetext{
* Coordinadora de la Maestría en Epidemiología y del Posdoctorado en Salud Colectiva de la Universidad Andina Simón Bolívar, Sede Ecuador.

** Docente de la Universidad San Francisco de Quito / Colectivo Espiral.

*** Docente de la Universidad de las Américas, Sede Ecuador / Colectivo Espiral.

**** Docente de la Universidad de las Américas, Sede Ecuador / Colectivo Espiral.

*****Docente del Programa Andino de Derechos Humanos de la Universidad Andina Simón Bolívar, Sede Ecuador.
} 
Portoviejo. In both cases, the accompaniment is aimed to the strengthening of the community organization, which reveals the perverse effects associated with disasters, such as land concentration or environmental discrimination. The phases: contact establishment, activities, methodology and conclusions are described, from a perspective of collective health and human rights.

KeYwords: earthquake Ecuador, Manabí, psychosocial accompaniment, collective health, human rights.

\section{INTRODUCCIÓN}

$\mathrm{E}$ 1 terremoto del 16 de abril de 2016 presentó una magnitud de 7,8 en la escala de Richter, devastó la provincia de Manabí y afectó a otras cuatro, especialmente Esmeraldas. Manabí, tradicionalmente ligada a la pesca, la agricultura o la exportación de productos artesanales, ha presentado históricamente un proceso de pérdida de identidad comunitaria que se ha profundizado tras el terremoto.

Así, se desorganizó una situación ya precaria de exclusión y pobreza, poniendo al desnudo una verdad oculta: el 42,3\% de la población vive bajo la línea de pobreza, el $74,4 \%$ no cuenta con acceso a agua potable, el $60 \%$ de la población urbana no dispone de servicio de alcantarillado, y únicamente el 15\% cuenta con servicio de recolección de residuos. ${ }^{1}$

Bajo este contexto, se presentan dos experiencias de acompañamiento psicosocial realizadas en los meses posteriores al terremoto, con el objetivo de fortalecer la organización comunitaria para abordar algunas de las problemáticas de modo cohesionado. Para esto, se trabajó en dos barrios: en primer lugar se describirá el acompañamiento realizado en el barrio Ébano en Jama, y a continuación en el barrio los Cañonazos en Portoviejo. En ambos casos se describen los daños observados, el contacto establecido con las comunidades y las evaluaciones realizadas, bajo una metodología común de la Investigación Acción Participativa (IAP).

1. Senplades 2015. Rendición de cuentas 2014. Disponible en «http://www.planificacion.gob.ec/wp-content/ uploads/downloads/2015/04/Informe-final-de-rendici\%C3\%B3n-de-cuentras-Zona-5-.pdf〉. 
Las experiencias describen, asimismo, las actividades que se orientan a la reparación de derechos vulnerados, y, en la última parte, se analizan algunos principios orientados a la reparación integral en personas y comunidades afectadas por desastres.

\section{EXPERIENCIA 1: ACOMPAÑAMIENTO PSICOSOCIAL ${ }^{2}$ EN EL BARRIO ÉBANO, JAMA}

El barrio Ébano de Jama constituye una de las poblaciones más cercanas al epicentro del terremoto, y ya presentaba complejas condiciones sociales preexistentes. ${ }^{3} \mathrm{El}$ cantón alberga 25.448 personas, siendo el $72 \%$ rural, y, según el INEC, se presentan altos índices de pobreza por necesidades básicas insatisfechas (NBI): 90,3\%, e índices de pobreza por consumo, $56 \%{ }^{4}$

En el terremoto fallecieron veintiocho personas, y respecto a pérdidas económicas, se reportaron que, de un total de 1.449 viviendas, 940 fueron investigadas en el Registro Único de Damnificados, siendo 863 calificadas como damnificadas. ${ }^{5}$ Se levantaron un total de 17 albergues y refugios, que un año después del terremoto ya habían sido cerrados.

La intervención del Colectivo Espiral ${ }^{6}$ se organizó bajo un modelo de trabajo comunitario que no buscaba únicamente cubrir necesidades inmediatas (que fueron demandadas por los miembros del barrio), sino que también procuró trabajar con la vul-

2. Entendemos el acompañamiento psicosocial como un proceso respetuoso, horizontal, ampliamente participativo en el que el profesional trabaja por la recuperación y reparación de las afectaciones en las dimensiones social, cultural, psicoafectiva (sentimientos), psicosomática (manifestaciones físicas de daño) y cognitiva (en los mecanismos de comunicación y afrontamiento); comprendiendo que estas afecciones son procesos dotados de historicidad y territorialidad, enmarcados en una conflictividad social estructural y que se expresan a nivel de los individuos, sus familias y sus grupos sociales.

3. De acuerdo con el Instituto Nacional de Estadística y Censos (INEC), en el cantón se presentan múltiples necesidades básicas: un 13,3\% no tiene acceso a la luz eléctrica, un 10,5\% de las viviendas no disponen de acceso a la red de alcantarillado, hay un 43,3\% de hacinamiento, y un 5,6\% de los hogares tienen niños y niñas que no asisten a la escuela. Instituto Nacional de Estadística y Censos, Censo de Población y Vivienda 2010 (Quito: INEC, 2010).

4. Andrea Molina et al., Mapa de pobreza y desigualdad por consumo Ecuador 2014 (Quito: INEC-BM, 2015), 87.

5. Brenda Sempértegui et al., Reconstruyendo las cifras luego del sismo. Memorias (Quito: INEC, 2017), 18-84.

6. El Colectivo Espiral es un grupo interdisciplinario integrado por profesionales psicólogos, educadores y médicos voluntarios, que se conformó espontáneamente tras el terremoto. 


\section{Mapa 1. Acciones realizadas en el Barrio Ébano, Jama}

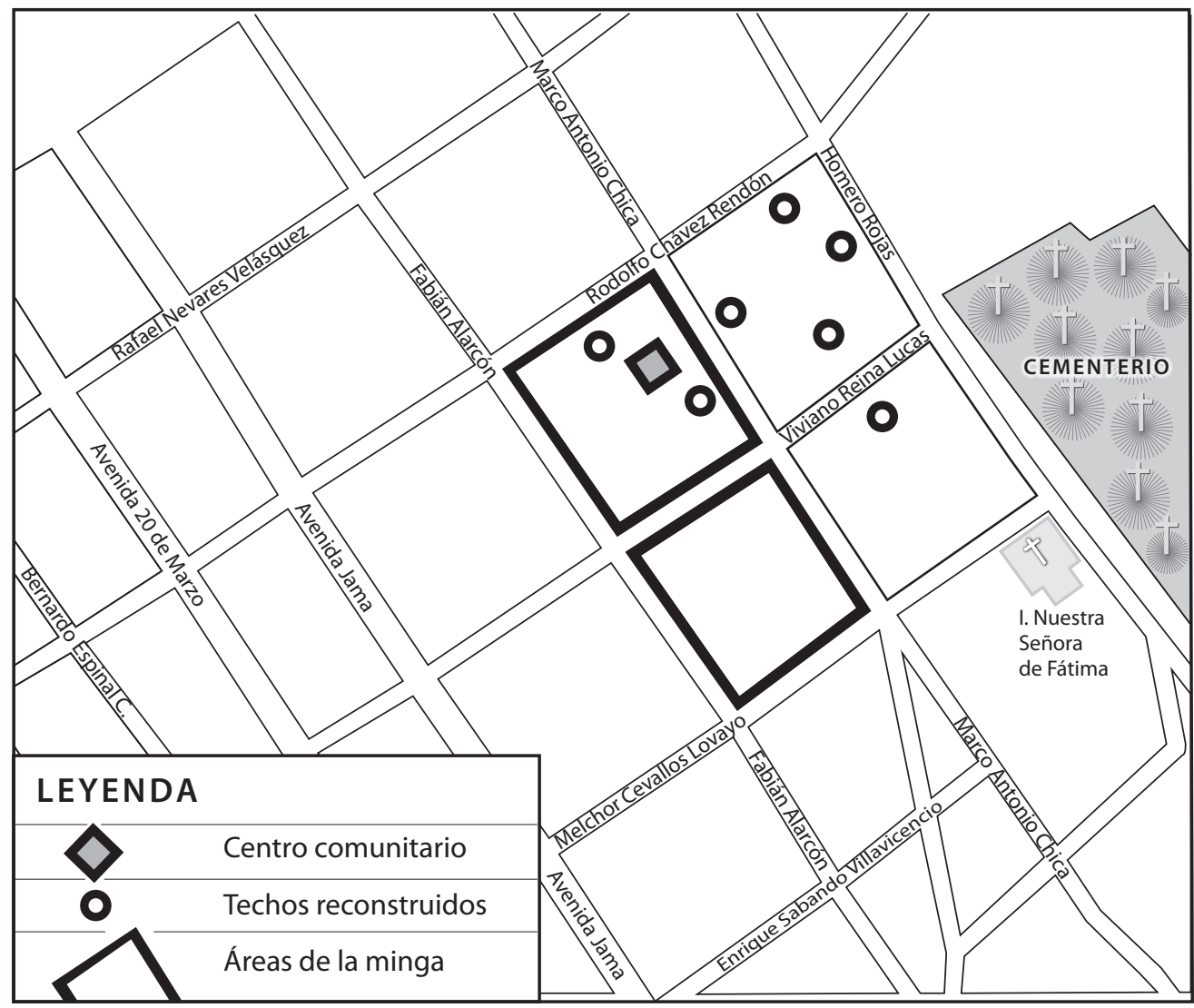

nerabilidad social, motivacional y física del entorno, ${ }^{7}$ evidenciando las capacidades de la propia población para generar cambios en su comunidad.

Las actuaciones en el barrio Ébano se focalizaron en el centro comunitario, que se convirtió en un espacio de reunión para las diferentes actividades que trataremos posteriormente. En el mapa 1 se observan las distintas zonas de acción, una de las cuales implica la reconstrucción de diferentes techos en el barrio, incluyendo el centro comunitario, y una minga en las áreas señaladas en las que los vecinos ayudaron a la limpieza del barrio.

7. Pau Pérez-Sales, "Intervención en catástrofes desde un enfoque psicosocial y comunitario", Átopos, No. 1 (2004): 5-16. 
El acompañamiento comenzó un mes después del terremoto, enfocándose en los impactos comunitarios, procurando formar espacios de encuentro entre hombres, mujeres, niños, adolescentes y adultos mayores, tratando la vivencia individual y grupal posterior a la catástrofe. Esto implicó un reconocimiento de los miembros como agentes de intervención, con quienes se generaron en conjunto cambios dentro del entorno. ${ }^{8}$ De esta manera, el acompañamiento psicosocial del Colectivo Espiral asumió una postura horizontal, que permitió vivenciar las experiencias de cada integrante del barrio, considerándolos como sujetos activos y no como objetos de intervención. ${ }^{9}$ Este acompañamiento psicosocial en el barrio Ébano se orientó en cuatro aspectos: a) acompañamiento a grupos vulnerables; b) proceso de diagnóstico comunitario y conformación de directiva; c) actividades comunitarias relevantes; y d) gestión de ayudas externas.

\section{a) Acompañamiento a grupos de atención prioritaria}

Entre las actividades generadas a partir del diálogo con los miembros del barrio, se conformó un trabajo con dos grupos: un espacio con niños y niñas de 4 a 12 años, y otro con mujeres.

\section{Niños y niñas}

El trabajo fue lúdico y estuvo vinculado al reconocimiento de emociones, al evidenciarse miedo e ira luego del desastre. En este punto se brindaron espacios para hablar del terremoto, reconocer e integrar emociones básicas. La capacidad para reconocer y categorizar expresiones faciales aparece en la infancia entre los 3 y 6 meses, y luego se presenta de forma más gradual entre los 5 y 7 años hasta la adolescencia. ${ }^{10}$ Dicho reconocimiento de emociones propias y ajenas constituye un proceso que permite discernir entre ellas, de tal forma que puedan generar empatía en los demás.

8. Catherine Walsh, edit., "Pedagogías decoloniales". En Prácticas insurgentes de resistir, (re) existir y (re) vivir (Quito: Abya-Yala, 2013).

9. Paula Yépez, Carolina Cedeño, Eduardo Granja y Tarquino Yacelga, "Aproximación a los enfoques de género e interseccionalidad: Aprendizajes significativos en la interacción de estudiantes universitarios con una comunidad indígena del Ecuador", Revista Internacional de Ciencias Sociales, No. 6 (2017): 67-79.

10. Javier Celdrán y Carmen Ferrándiz, "Reconocimiento de emociones en niños de Educación Primaria: Eficacia de un programa educativo para reconocer emociones", Electronic Journal of Research in Educational Psychology, No. 10 (2012): 1321-42. 


\section{Mujeres}

El acompañamiento en este grupo consideró el enfoque de género como un eje fundamental para descubrir cuánto de arbitrario hay en la posición que mujeres y varones ocupan en la sociedad. ${ }^{11}$ Las mujeres después del terremoto han sido visibilizadas como un eje de cohesión, nutricio y de conocimiento de los entornos comunitarios, a pesar de que no necesariamente configuran un liderazgo. Al respecto, se decidió conformar un grupo de mujeres para conocer sus necesidades y fomentar un espacio de encuentro y escucha en el que pudieran expresar ideas y sentimientos. El grupo estaba conformado por integrantes de entre 12 y 70 años. Se compartieron habilidades y conocimientos de cada una, así como estrategias de fortalecimiento del grupo y del barrio.

\section{b) Proceso de diagnóstico comunitario y conformación de una directiva comunitaria}

Este proceso se inició con el reconocimiento de las necesidades, los cambios y conflictos internos. La metodología utilizada fue la cartografía social, técnica participativa que permite identificar información sobre un territorio social de problemas, redes y la dinámica comunitaria. ${ }^{12}$ Luego, se generó la discusión de los límites espaciales del barrio, que permitió identificar la falta de espacios comunitarios y, por ende, de actividades colectivas. Asimismo, se identificaron reacciones de incertidumbre y desamparo, pues percibieron que no son escuchados ni atendidos por instituciones públicas. Además, describieron la ausencia de habitantes que abandonaron el sector y una alta preocupación a nivel laboral, ya que la actividad económica se paralizó, se observó escasa organización y convocatoria en adultos y conflictos informales entre vecinos. Dispusieron de escasa infraestructura, compuesta por material de plásticos y carpas temporales, que les produjo inseguridad y vulnerabilidad.

Ante esto, se buscó construir en conjunto soluciones que ayuden a enfrentar los problemas personales y colectivos posterremoto. A partir de lo anterior, se propone la generación de espacios en los cuales puedan trabajar lazos comunitarios y, luego, un trabajo organizativo.

11. Claudia Korol, comp., Hacia una pedagogía feminista. Géneros y educación popular (Buenos Aires: El Colectivo, 2007), 11.

12. Sabina Habegger y Iulia Mancila, "El poder de la cartografía social en las prácticas contrahegemónicas o la cartografía social como estrategia para diagnosticar nuestro territorio", Revista Araciega, No. 14 (2006). 
Así, surgió la necesidad de conformar una directiva barrial que los represente. Se promovió un recuento de los hechos relacionados con el terremoto y sus consecuencias, así como de los compromisos adquiridos en reuniones comunitarias. Luego se plantearon algunas preguntas con el fin de conocer sus percepciones sobre la pertinencia de una directiva barrial con el fin de explorar su compromiso. Las respuestas se describen en la tabla 1 .

Tabla 1. Proceso de conformación de una directiva en barrio Ébano

\begin{tabular}{|l|l|}
\hline \multicolumn{1}{|c|}{ Preguntas orientadoras } & \multicolumn{1}{|c|}{ Respuestas de la comunidad } \\
\hline $\begin{array}{l}\text { 1. ¿Para qué una directiva } \\
\text { en el barrio Ébano? }\end{array}$ & $\begin{array}{l}\text {-Para tener información sobre la llegada de asistencia material. } \\
\text {-Para facilitar la gestión de las ayudas (buscar un espacio, limpiar la ruta } \\
\text { de evacuación, exigir apoyo médico y de profesionales). } \\
\text {-Para representar y conocer las necesidades del barrio. } \\
\text {-Para organizarse y comunicarse mejor, fortalecer la unión. }\end{array}$ \\
\hline $\begin{array}{l}\text { 2. ¿Cómo involucrarnos } \\
\text { con la directiva? }\end{array}$ & $\begin{array}{l}\text {-Comunicarse y pasar la voz. } \\
\text {-Estar unidos y ser colaboradores. } \\
\text {-Ser participativos, para que no toda la responsabilidad sea de la directiva. } \\
\text {-Generar responsabilidad colectiva. }\end{array}$ \\
\hline
\end{tabular}

Fuente y elaboración: los autores, 2017.

Paralelamente a esta actividad se identifican tres factores sobre la directiva barrial: a) manejo del dinero, que describe necesidades de gestión e imprevistos, pero que debe contar con un reporte de gastos; b) tiempo de duración, se plantea un año de duración como mínimo; y c) número de personas, en el que se identifican siete directivos como mínimo, que incluye los cargos de presidente, vicepresidente, tesorero, secretario, y tres vocales que tendrán la función de coordinadores de actividades específicas.

\section{c) Actividades comunitarias relevantes: minga-cine-festejos}

La primera acción de la directiva comunitaria fue llevar a cabo una minga para alcanzar un beneficio equitativo de los productos o del entorno. ${ }^{13}$ El objetivo era reunir a la colectividad en un esfuerzo de mejora del barrio, actividad que se realizó el 11 de

13. Pascual Yépez, "Tradiciones indígenas en el mundo moderno y su impacto en la educación intercultural", SOPHIA, Colección de Filosofia de la Educación, No. 18 (2015): 231-51. 
septiembre de 2016. Luis, presidente de la directiva, informó de la participación de aproximadamente 20 vecinos, en un trabajo que resultó satisfactorio y ameno.

Por otra parte, el cine-foro se centró en películas que favorecían el reconocimiento de emociones, como Intensamente (Inside Out), Wall-E, o películas educativas tales como Yo soy Sam y Cómo entrenar a tu dragón. El cine fue usado para reforzar conceptos y, tras la proyección, compartir impresiones con los asistentes, especialmente con niños y niñas.

Los festejos se realizaron como espacios de conmemoración y celebración: por el Día del Niño al inicio del proyecto, en el cual se realizó una gymkana para fortalecer el trabajo en equipo; y en Navidad, como cierre del mismo, el cual fue implementado por los propios pobladores del barrio y la directiva. La comunidad organizó música, baile y agasajo con comida y se proyectaron dos videos, uno realizado por el Colectivo Espiral sobre las diferentes actuaciones, y otro enviado desde España por la ONG Corazón del Mundo, que apoyó económicamente algunas iniciativas para el barrio.

\section{d) Gestión de ayudas externas: ONG y médicos}

A raíz de los daños ocasionados por el terremoto y el fenómeno de El Niño durante el invierno de 2017 decidimos impulsar una iniciativa de inversión, en esta ocasión con la idea de construir ocho techos definitivos en estructuras que estaban seriamente dañadas a causa de la crudeza de la época de lluvias. Las siete familias seleccionadas estaban entre las más necesitadas del barrio, ya que por el terremoto habían perdido sus casas y las estructuras temporales construidas perdieron los techos de lona. Las personas estaban en alto riesgo por su avanzada edad, familias con varios hijos o monoparentales, por lo que se reconstruyeron ocho techos, incluyendo un espacio que se utiliza para reuniones y talleres comunitarios. El proyecto fue realizado por Embudo, al frente del cual estuvo Begoña Izquierdo y el Colectivo Espiral. Además, colaboraron las ONG españolas Corazón del Mundo, ADAPA y personas a título individual, los cuales financiaron los materiales. Embudo canalizó a los voluntarios para la instalación de los techos en estructuras que ellos mismos habían impulsado poco después del terremoto.

Otro aporte fue de profesionales en medicina, para realizar chequeos médicos básicos dirigidos hacia adultos mayores, quienes presentaban enfermedades crónicas tales como hipertensión y diabetes. Estas personas no habían acudido al centro de salud en Jama por temor a un nuevo terremoto, ya que este centro de salud presentaba huellas superficiales de lo ocurrido. También se realizó un taller de sexualidad enfocado en la prevención de embarazo adolescente. Finalmente, se gestionó la recepción de una donación de material escolar proveniente de Bélgica. 


\section{REFLEXIONES SOBRE EL ACOMPAÑAMIENTO EN EL BARRIO ÉBANO}

Las diversas acciones que se realizaron desde el Colectivo Espiral se orientaron al respeto de la identidad comunitaria que busca consensos respecto a cómo minimizar los impactos del terremoto. En este sentido, se reconoce que la metodología de acompañamiento psicosocial distingue procesos y grupos, los cuales no siempre van en línea con una propuesta externa. En ocasiones, las personas afectadas esperaban un apoyo concreto, fundamentalmente ayudas materiales, lo cual configura un desafío el establecer y trabajar formas alternativas que se orienten a una contención emocional, proporcionar información, promover la organización comunitaria y gestionar recursos.

En este punto, la diversidad de profesionales y la realización de distintas actividades favoreció la recepción en los integrantes del barrio. Por otro lado, se intentó fortalecer las capacidades de sus integrantes, ya que habitualmente en estos contextos posdesastre, las comunidades esperan que sean sus líderes los que gestionen la ayuda externa. El camino recorrido con los habitantes del barrio Ébano ejemplifica que las acciones solidarias de grupos o asociaciones externas deben responder a las necesidades colectivas de las poblaciones afectadas por desastres, muchas de las cuales no son explícitas, que requieren de tiempo para ponerlas de manifiesto y que la propia comunidad vea y valore su pertinencia.

\section{EXPERIENCIA 2: ACOMPAÑAMIENTO PSICOSOCIAL EN EL BARRIO LOS CAÑONAZOS, PARROQUIA SAN PABLO, PORTOVIEJO}

El barrio Los Cañonazos, ubicado en la parroquia urbano marginal San Pablo, constituye un territorio que históricamente ha sufrido discriminación social y ecológica. Viven aproximadamente 200 familias, $80 \%$ de las cuales se dedicaban hasta febrero de 2017, al reciclaje a cielo abierto, es decir, trabajaban en condiciones de informalidad en el basural del cantón Portoviejo. Por un lado, recuperaban, clasificaban y comercializaban residuos inorgánicos, y, por otro, utilizaban los residuos orgánicos para la alimentación de animales domésticos.

El basural a cielo abierto del cantón Portoviejo es uno de los sistemas de disposición final más antiguos del Ecuador (50 años), y con mayor población recicladora viviendo dentro y en sus alrededores. Con una extensión de 14 hectáreas, el botadero se localiza a $3,5 \mathrm{~km}$ de la ciudad de Portoviejo, al término de la parroquia urbanomarginal San Pablo. La estimación del volumen promedio de residuos tratados en este cantón es de 300 toneladas diarias, de las cuales el 60\% está constituido por residuos 
orgánicos, 35\% inorgánicos, y el 5\% restante desechos tóxicos. La disposición final se realizaba en condiciones sumamente precarias, puesto que no existía separación en la fuente, por lo que los residuos se vertían mezclados, incluso lodos de pozos sépticos y residuos industriales.

Con el terremoto del 16 de abril de 2016, Portoviejo, especialmente su casco central, fue una de las ciudades más afectadas del país. Desde una visión crítica que problematiza la comprensión de los fenómenos naturales como desastres, entendemos que no existen desastres naturales; por el contrario, existen fenómenos naturales que se convierten en desastres por la pauperización, la inequidad social y ecológica, y relaciones de poder desiguales que fundan y sostienen profundas brechas entre la opulencia y la miseria.

Bajo este enfoque crítico, podemos entender la segregación histórico-territorial de los daños sufridos en las familias de Portoviejo. Mientras los barrios centrales que eran esencialmente barrios populares-comerciales desaparecieron, barrios de inserciones socioeconómicas medias altas y altas, sufrieron pocos daños. Sin embargo, el barrio Los Cañonazos, en la parroquia San Pablo, pese, o mejor aún, al ser uno de los barrios más excluidos y que durante décadas ha amortiguado los impactos ecológicos, económicos y sociales del basural a cielo abierto, resultó prácticamente inmune al terremoto. Solamente cuatro casas sufrieron afecciones graves y debieron ser reconstruidas, no se registraron muertos y los heridos reportaron afecciones físicas leves.

Sin embargo, el trauma psíquico estuvo presente especialmente en las primeras semanas y meses que registraron réplicas. En algunos casos la intensidad del daño psicoafectivo se expresaba también en condiciones de daño psicosomático (marca en el cuerpo) e incluso en alteraciones cognitivas (especialmente en algunos niños y niñas y en adultos mayores). La magnitud de los daños obligó a buscar opciones para la disposición de las toneladas de escombros que fueron removidas en las primeras semanas, y el basural a cielo abierto de Portoviejo se constituyó uno de los principales lugares seleccionados. Es así que durante los meses de mayo a septiembre de 2016, las familias recicladoras pudieron recuperar muchos de los materiales dispuestos, por sobre la disputa con las autoridades municipales que reclamaban propiedad sobre materiales de alto valor comercial como el cobre.

En este contexto, las familias del barrio Los Cañonazos tuvieron ingresos adicionales importantes durante los primeros cuatro meses posteriores al terremoto, situación que contrasta con el aviso de salida o desalojo que recibieron por parte del municipio de Portoviejo en el mes de noviembre. El cierre del basural a cielo abierto, con el argumento del inicio de un proyecto de relleno sanitario, sin recicladores, se programó para el 20 de febrero de 2017, siendo el terremoto uno de los elementos determinantes para esta transición. Con el cierre del basural, únicamente 20 familias fueron incorporadas en el proyecto piloto para la recolección y reciclaje en fuente, quedando 
aproximadamente 80 familias sin alternativas económicas y desatando una situación de conflictividad social estructural y disputa por derechos laborales y sociales.

En el marco de esta situación, retomamos los procesos de acompañamiento psicosocial, que no inician con el terremoto, sino que vienen de un histórico de 10 años con familias de recicladores y recicladoras.

\section{Aproximaciones metodológicas}

Desde un enfoque de salud colectiva, entendemos que el estado individual de fisiología/fisiopatología está determinado por tres niveles de la realidad: la realidad general (contextos histórico-territoriales), la realidad particular (los modos de vida de las comunidades, barrios y grupos sociales) y la realidad singular (los estilos de vida). Estos tres niveles operan en una suerte de relación dialéctica de interafección e interinfluencia. A su vez, estas estructuras sociales complejas sostienen relaciones dialécticas con la naturaleza de la que forman parte, en un proceso conocido como metabolismo social. De esta manera, al tiempo que los individuos organizados en sociedades, con sus modos de reproducción social y material, afectan a las naturalezas de las que forman parte, también son afectados por ellas.

Así, las naturalezas alteradas y los contextos histórico-territoriales opresores van a determinar las realidades particulares, es decir, los modos colectivos de vivir y finalmente los estilos de vida de las personas y sus condiciones de vulnerabilidad (procesos destructivos de la salud a nivel individual) y/o resiliencia (respuestas a procesos positivos y negativos a nivel individual). Los movimientos de interrelación entre estos tres dominios de la realidad son conocidos como movimientos de subsunción-autonomía relativa ${ }^{14}$ y determinan finalmente la encarnación, que es la expresión, en el cuerpo, de la exposición a esos procesos.

La operacionalización de la metodología, que se detalla en la tabla 2, se encuentra ajustada al movimiento dialéctico de la complejidad de los dominios: general, particular y singular, siendo las técnicas de investigación-intervención definidas en cada uno de estos tres espacios. Se considera, entonces, que las afecciones psico-emocionales descritas no pueden estudiarse aisladas del dominio particular (reproducción material -modelos productivos-; reproducción social -cuidado y crianza de la vida-; consumo; organización social; y relación con la naturaleza) y que, al mismo tiempo, este

14. Jaime Breilh, Epidemiología crítica: Ciencia emancipadora e interculturalidad, 2a. ed. (Buenos Aires: Lugar Editorial, 2004). 
dominio particular o de los modos de vida obedece y cambia en función del escenario geopolítico local, nacional, regional y global (dimensión o dominio general).

Tabla 2. Operacionalización metodológica

\begin{tabular}{|l|l|l|}
\hline \multicolumn{1}{|c|}{ Componente } & \multicolumn{1}{c|}{ Aproximación metodológica } & \multicolumn{1}{c|}{ Informantes } \\
\hline $\begin{array}{l}\text { Segregación histórico- } \\
\text { territorial del cantón Por- } \\
\text { toviejo }^{1}\end{array}$ & $\begin{array}{l}\text { Cartografía social, análisis de la or- } \\
\text { ganización sociohistórica del can- } \\
\text { tón Portoviejo }\end{array}$ & $\begin{array}{l}\text { Senplades } \\
\text { Levantamiento de información } \\
\text { geográfica in situ }\end{array}$ \\
\hline Modos de vida barrial $^{\text {Etnografía a cinco Asambleas ba- }}$ & $\begin{array}{l}\text { Autoridades municipales } \\
\text { Pobladores del barrio Los Cañona- } \\
\text { zos }\end{array}$ \\
\hline $\begin{array}{l}\text { Salud individual y fami- } \\
\text { liar }\end{array}$ & Acompañamiento psicosocial & $\begin{array}{l}\text { Familias que solicitan acompaña- } \\
\text { miento }\end{array}$ \\
\hline
\end{tabular}

1. Desde la Ecología Política, entendemos el territorio como un espacio socioecológico vivo en el que grupos sociales organizados, con sus formas de producción, reproducción social, con unos modos de consumo, una vida cultural y construcciones identitarias particulares, transforman sus naturalezas y en ese proceso también son transformados por ellas; así, la segregación histórico territorial comprende el análisis de las trasformaciones de estos territorios en el tiempo, de los grupos sociales que los constituyen, de las relaciones de poder y los procesos protectores y destructivos que operan a este nivel.

Fuente y elaboración: los autores, 2017.

\section{ReSUltados y HALlaZgos: \\ RECUPERANDO LA SALUD, ACOMPAÑAMIENTO PSICOSOCIAL}

\section{A nivel territorial}

Las metodologías trabajadas a nivel barrio-territorio se centraron en el análisis colectivo de la segregación del daño en el cantón Portoviejo. En asamblea comunitaria, revisamos la proyección de varios mapas realizados por el Área de Salud de la Universidad Andina Simón Bolívar, Sede Ecuador, que demostraban la afección diferencial del terremoto, por inserción social y ecológica.

Este trabajo de reflexión-discusión nos permitió comprender de qué forma los procesos históricos de exclusión social y ecológica que habían determinado la ubicación del barrio Los Cañonazos en una zona marginal y próxima al basural a cielo abierto resultaron en un proceso protector de la vida. El tipo de construcción de las viviendas, elaborados de caña, fue determinante del mantenimiento casi intacto de la infraestructura del barrio. El análisis de territorio, por un lado, tranquilizó a las familias frente a las réplicas, en tanto existía claridad en relación a lo que se podría esperar del daño y afecciones. Por otro lado, permitió la toma de decisiones para la potenciación de 
Mapa 2. Infraestructura afectada y precariedad de la vivienda

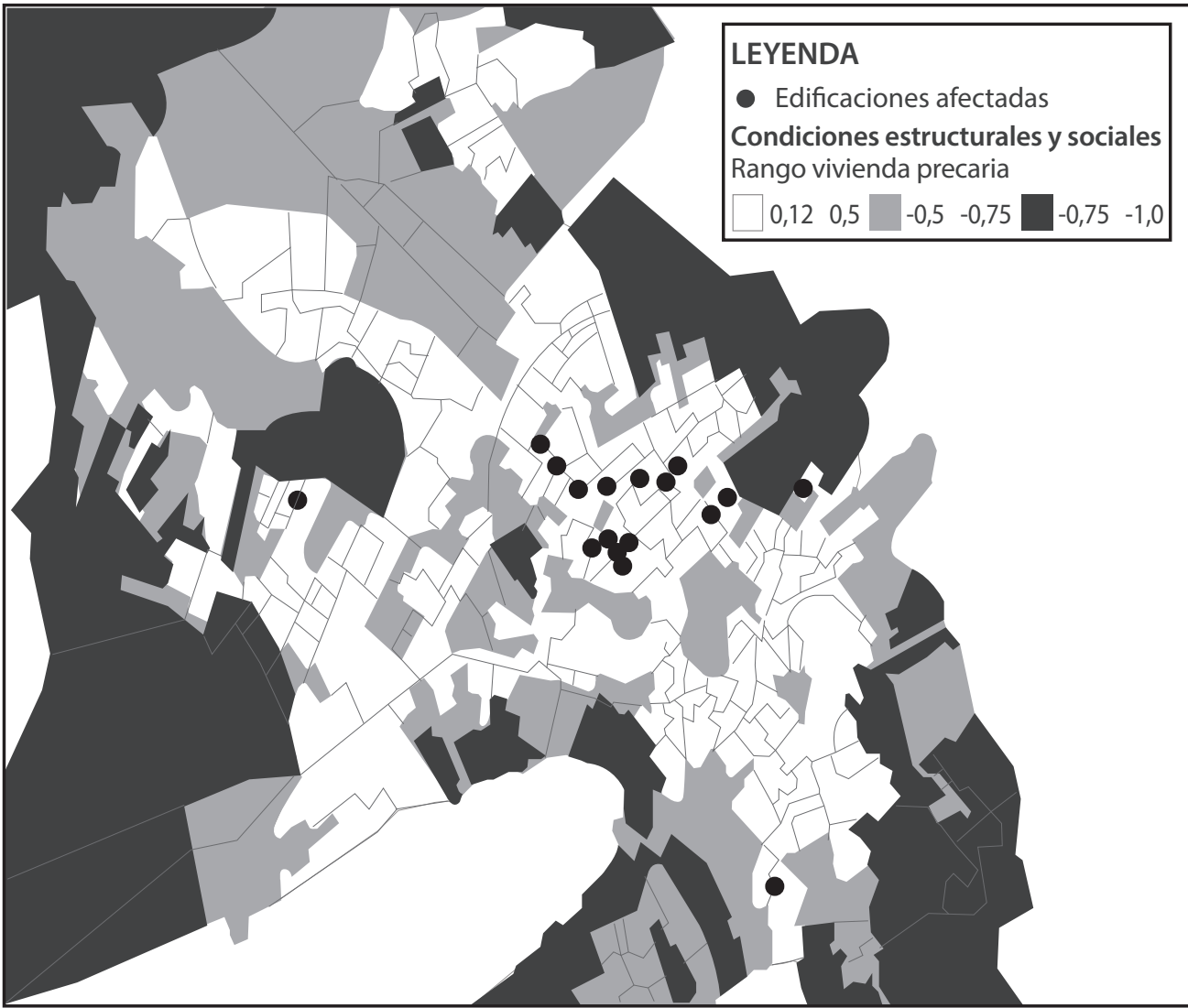

Fuente y elaboración: Jaime Breilh y Gianina Zamora, Área de Salud, Universidad Andina Simón Bolívar, 2016.

procesos protectores de la salud y la vida, y el control de los procesos destructivos de la salud y la vida. El barrio se organizó realizando recorridos participativos para el fortalecimiento de las estructuras de caña, la identificación de las infraestructuras precarias de cemento y la reconstrucción de las casas caídas, priorizando materiales como la caña y la madera.

\section{A nivel familiar-barrial}

Este campo se trabajó a través de observación participante (etnografía) y asambleas comunitarias, primero quincenal y luego mensualmente. Las asambleas se rea- 
Mapa 3. Afecciones en el barrio Los Cañonazos

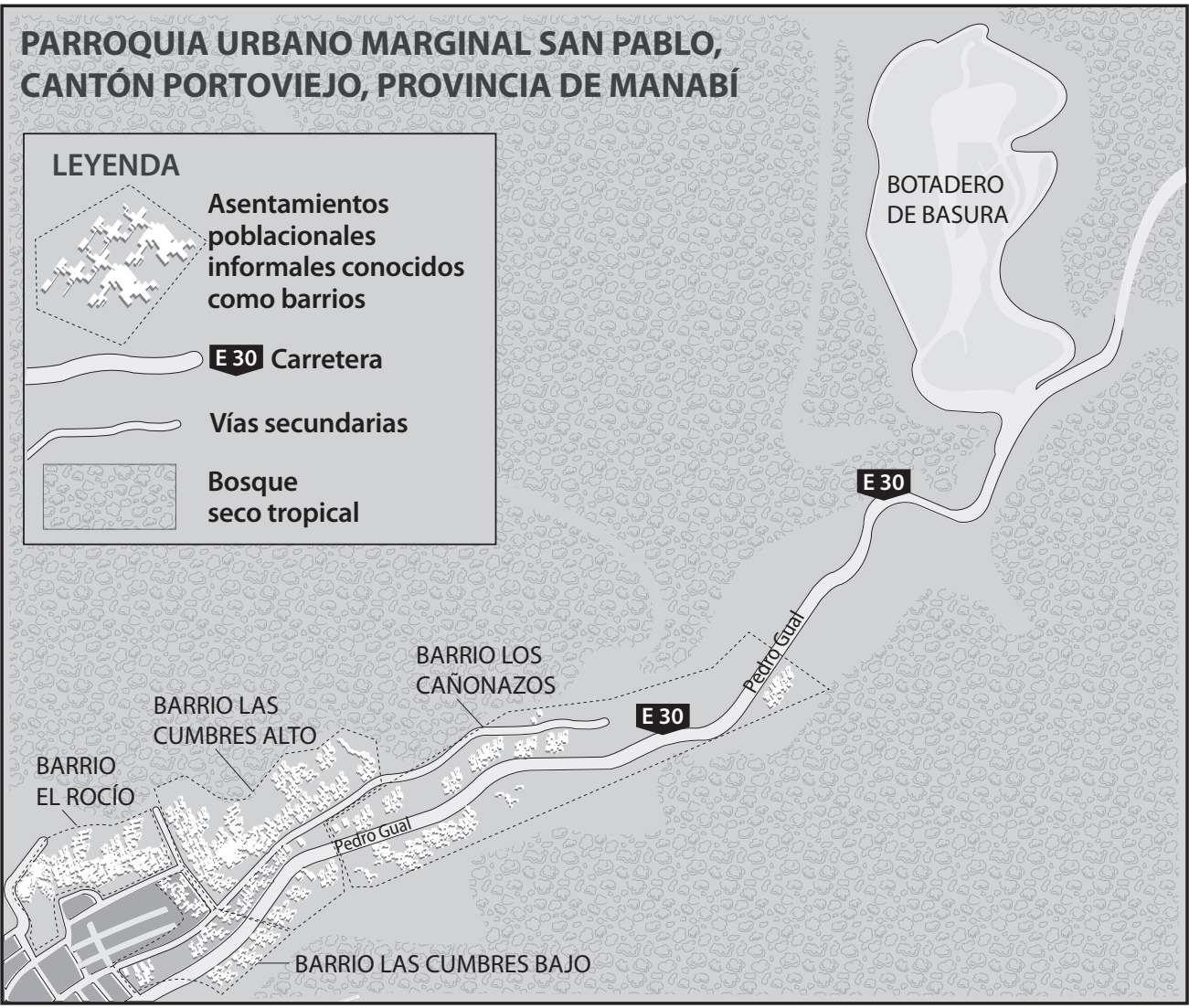

Elaboración: Raúl Yépez.

lizaron con las familias de la asociación de recicladores y se orientaron a analizar de qué forma el terremoto había afectado las cinco dimensiones de los modos de vida comunitarios:

a) Los modos de producción: se evaluó de qué forma la disposición de escombros en el basural a cielo abierto estaba constituyendo una oportunidad de ingresos económicos adicionales por la recuperación de materiales especialmente metálicos. No obstante, imponía nuevos procesos destructivos de la salud tales como la presencia de restos humanos, el incremento de transporte pesado en las zonas en las que los recicladores trabajaban diariamente, entre otras. El análisis se proyectó a los siguientes meses, con la claridad de que cada vez llegaban menos materiales, y, finalmente, con el aviso de desalojo. La preocupación por 
el desalojo intensificó el daño psicosocial, en tanto se trataba de anular una actividad productiva que había sostenido durante al menos cuatro generaciones a estas familias. Hasta el día de hoy, algunas familias siguen viviendo dentro del basural, y, pese a lo autoritario de la medida, se niegan a dejar su trabajo, ingresan clandestinamente desde tierras forestales, caminando durante horas, en la noche y madrugada para seguir recuperando materiales dentro del basural. Se han reportado enfrentamientos violentos entre grupos de recicladores y fuerzas policiales municipales y nacionales.

Como se describió, 20 familias se han mantenido en el proyecto piloto para la recuperación domiciliar en un barrio de la ciudad. En este sentido, parte del fracaso de esta transición se debe a que el municipio del cantón no ha provisto de condiciones materiales reales a los recicladores para iniciar esta transición, no tienen triciclos para trasladar el material, no tienen centros de acopio y solo disponen de una ruta para el trabajo.

b) Reproducción social: el cuidado, crianza y trabajo doméstico en las familias ha estado históricamente relegado a las mujeres. Es así que, a raíz del terremoto, las mujeres manifiestan que se encuentran sobrecargadas, el daño psicoafectivo se intensifica en condiciones de intenso estrés laboral. El 67\% de las mujeres recolectoras presenta un nivel severo de estrés laboral, en comparación con el $18,8 \%$ de los varones dedicados a la misma actividad. Asimismo, el 70,8\% de las mujeres reporta sufrimiento mental severo, y el $81 \%$ de ellas son recolectoras. Mientras los varones han optado por el consumo de drogas que los mantiene ajenos a la realidad, las mujeres naturalizan y normalizan las condiciones precarias de vida, acumulando todos los conflictos, presiones y temores. Las mujeres manifiestan que sus parejas gastan el dinero en actividades recreativas que realizan luego del reciclaje, mientras que ellas deben encargarse, además del trabajo, de las actividades de cuidado y crianza. En consecuencia, una de las propuestas asamblearias fue el fortalecimiento de los circuitos de apoyo y recreación para mujeres.

c) Consumo: el incremento de los ingresos económicos durante los primeros cuatro meses posteriores al terremoto, por un lado, devino en un incremento del consumo de alcohol y drogas, especialmente en recicladores varones y jóvenes. Muchas de las familias aprovecharon ese ingreso adicional para la compra de celulares y televisores modernos y algunas mejoraron la infraestructura de su casa. Se trató de un pequeño boom que no tuvo implicaciones estructurales en el mejoramiento de la vida, inserción social y económica de las familias. Todos estos elementos fueron problematizados durante las sesiones asamblearias.

d) Organización social y de la cultura: si bien al inicio del terremoto se retomó y fortaleció la organización social de la asociación de recicladores, la presencia 
de las familias en las reuniones y asambleas no fue permanente: oscilaban entre 40-50 familias en el mejor de los casos, y en ocasiones 10 a 20 familias. La organización de la asociación se vio fortalecida frente al aviso de desalojo; sin embargo, finalmente fueron aproximadamente 20 a 30 familias las que se incorporaron al proceso de trabajo y transición al proyecto piloto para la recolección en fuente.

e) Relaciones con la naturaleza: indudablemente, el terremoto cambió las relaciones con la naturaleza, imponiendo lógicas de temor, pero también de reconocimiento de las relaciones dialécticas entre los grupos sociales y sus entornos ecológicos. Varias reflexiones se realizaron desde aproximaciones de Ecología Política que fortalecieron la comprensión y apropiación del territorio no como elementos externos sino como construcción social y material de la que somos parte.

\section{A nivel familiar-individual}

Con las familias que remitían condiciones importantes de afección psicoafectiva, se desarrollaron procesos de acompañamiento psicosocial en sus casas, con todos los miembros que querían participar, y cuando fue necesario se trabajó individualmente con los más afectados. Se realizaron libretas de sueños y miedos en las que cada familia dibujaba o escribía, registros que se trabajan quincenalmente. Con los niños y niñas se trabajaron metodologías lúdicas y gráficas y se sostuvieron procesos de alegremia. ${ }^{15}$ Con las mujeres se realizaron reuniones y talleres lúdico-testimoniales y grupos de apoyo, y con los adultos mayores se trabajó en escucha y masaje-terapia.

\section{REFLEXIONES SOBRE EL ACOMPAÑAMIENTO EN EL BARRio Los Cañonazos}

La continuidad de los procesos de IAP desarrollados posterior al terremoto se orientaron al fortalecimiento de procesos asociativos, de formación política y organización social, al tiempo que permitieron acompañar a familias que manifestaban afecciones psico-emocionales, psicosomáticas y psicosociales, estado de alerta exagerado e incapacidad de continuar con la vida cotidiana. El diseño, aspiraciones, objetivos, estrategias y funciones de este trabajo se definieron en reuniones con la Asociación

15. Su creador Julio Monsalvo la define como alegría circulado por la sangre. Para él, la alegremia significa estar sanos, y encontrarse con las siete "A": agua, aire, alimento, arte, amor, albergue, aprendizaje. 
de recicladores(as) y en Asambleas Comunitarias. Los productos derivados de este proceso fueron varios: agendas de compromisos comunitarios, el fortalecimiento de la Asociación de recicladores(as), informes científicos, publicaciones populares, intercambios, entre otros.

En este contexto, el presente artículo pretende denunciar las condiciones de opresión en las que viven los(as) recicladores(as) informales, condiciones que se hicieron particularmente visibles tras el terremoto. Esto abre el debate público sobre la urgencia de establecer políticas de reparación integral en comunidades afectadas por la disposición final de residuos.

\section{A MODO DE CONCLUSIÓN: ACOMPAÑAMIENTO Y REPARACIÓN INTEGRAL CON UN ENFOQUE DE DERECHOS HUMANOS}

El enfoque de Derechos Humanos describe una perspectiva que se centra en la población afectada por el terremoto del 16A que presenta desprotección, marginación, exclusión y/o discriminación, producto de vulneraciones a los derechos humanos. En este caso, los impactos que se generan en determinados grupos de población no son considerados efectos de un desastre natural, sino que, con anterioridad, no estaban provistas las condiciones que aseguraran que estos colectivos tuvieran acceso a derechos tales como vivienda digna, educación, salud o todos los relativos a los derechos económicos, sociales y colectivos. Esto a su vez condiciona que los procesos de recuperación posteriores al desastre se orienten al logro de una reparación integral, donde el Estado se responsabiliza en su acción vulneradora y reparadora.

Los procesos de acompañamiento psicosocial como los descritos en estas experiencias configuran dinámicas que se orientan a una reparación integral en algunos de los derechos vulnerados para estas comunidades. Según Wilches-Chaux, ${ }^{16}$ es posible identificar distintos derechos que se producen en contexto de desastre, ${ }^{17}$ que, bajo lo presentado en los barrios de Ébano y Los Cañonazos, pueden trabajarse desde grupos externos, no obstante representa una obligación del Estado.

16. Gustavo Wilches-Chaux, "Derechos de personas y comunidades afectadas por desastres", Coyuntura Política, No. 3 (1999).

17. El autor identifica nueve siguientes derechos: 1. Derecho a la protección del Estado; 2. Derecho a la información; 3. Derecho a la participación; 4. Derecho a la autogestión; 5. Derecho de prioridad; 6. Derecho a la continuidad de los procesos; 7. Derecho frente a los medios de comunicación; 8. Derecho a la prevención; 9. Derecho a la integralidad de los procesos. Un décimo derecho que se propone aquí es el derecho de las personas afectadas a recibir una atención integral en salud y salud mental. 
Así, el acompañamiento psicosocial de ambas experiencias se enmarca en el desarrollo de procesos que se caracterizan por incorporar:

a) La identificación de derechos vulnerados en las poblaciones afectadas, algunos de los cuales se relacionan con un antes y después del desastre.

b) Una aproximación territorial y compleja desde los barrios, que identifica además las necesidades de las personas, sus familias y sus grupos sociales.

c) Una orientación metodológica bajo el enfoque de la investigación acción participativa, que, por un lado, asume a la participación como un derecho, un deber y un mecanismo; y, por otro, busca llegar a la praxis o acción transformadora.

d) Una reflexión permanente en conjunto sobre las actividades y los procesos entre la comunidad y los equipos de acompañamiento.

Finalmente, las experiencias descritas en este texto presentan un cierre, confirmando que los procesos de acompañamiento configuran acciones temporales que potencian la organización comunitaria. Consideramos que el camino hacia la reparación integral, como obligación del Estado, requiere incluir una perspectiva amplia desde distintas disciplinas. Asimismo, resulta indispensable que los profesionales y/o integrantes de instancias académicas planifiquen y asuman una posición crítica tanto conceptual como metodológica en toda acción de acompañamiento que se oriente a la reparación integral de comunidades afectadas por desastres.

\section{BIBLIOGRAFÍA}

Breilh, Jaime. Epidemiología crítica: ciencia emancipadora e interculturalidad. 2a. ed. Buenos Aires: Lugar Editorial, 2004.

Celdrán Baños, Javier, y Carmen Ferrándiz García. "Reconocimiento de emociones en niños de Educación Primaria: Eficacia de un programa educativo para reconocer emociones". Electronic Journal of Research in Educational Psychology, No. 10 (2012).

Fernández, Itziar, Francisco Morales y Fernando Molero. Psicología de la intervención comunitaria. Bilbao: Desclée De Brouwer, 2011.

Habegger Sabina, e Iulia Mancila. "El poder de la cartografía social en las prácticas contrahegemónicas o la cartografía social como estrategia para diagnosticar nuestro territorio". Revista Araciega, No. 14 (2006).

Instituto Nacional de Estadística y Censos. Censo de Población y Vivienda 2010. Quito: INEC, 2010.

Korol, Claudia, comp. Hacia una pedagogía feminista. Buenos Aires: El Colectivo, 2007.

Matter, Anne, Martin Dietschi y Chrsitian Zurbrügg, "Improving the Informal Recycling Sector through Segregation of Waste in the Household. The case of Dhaka Bangladesh", $\mathrm{Ha}$ bitat Int., No. 38 (2013). 
Medina, Martin. "Serving the unserved: Informal Refuse Collection in Mexican Cities". Solid waste collection that benefits the urban poor. CWG Workshop, Dar es Salaam, Paper No. 17, Tanzania, 2003. Disponible en 〈http://journals.sagepub.com/ doi/10.1177/0734242X05057698>.

Molina, Andrea, et al. Mapa de pobreza y desigualdad por consumo Ecuador 2014. Quito: INEC-BM, 2015.

Montero, Maritza. Introducción a la psicología comunitaria: desarrollo, conceptos y procesos. Buenos Aires: Paidós, 2004.

Pérez-Sales, Pau. "Intervención en catástrofes desde un enfoque psicosocial y comunitario". Átopos, No. 1 (2004).

Sempértegui, Brenda, et al. Reconstruyendo las cifras luego del sismo. Memorias. Quito: INEC, 2017.

Solíz, Fernanda. Salud colectiva y ecología política: La basura en Ecuador. Quito: Universidad Andina Simón Bolívar, Sede Ecuador / La Tierra, 2015.

—, y Adolfo Maldonado. Guía de metodologías comunitarias participativas. Quito: Clínica Ambiental, 2010.

Van Eerd, Dwayne. The Occupational Health Aspects of Waste Collection and Recycling. A Survey of the Literature. Toronto: UWEP, 1996. Disponible en 〈http://docs.watsan.net/ Downloaded_Files/PDF/Eerd-1996-Occupational〉.

Walsh, Catherine, comp. Pedagogías decoloniales. Prácticas insurgentes de resistir, (re)existir $y$ (re)vivir. Quito: Abya-Yala, 2013.

Wilches-Chaux, Gustavo. "Derechos de personas y comunidades afectadas por desastres". Coyuntura Política, No. 3 (1999).

Yépez, Pascual. "Tradiciones indígenas en el mundo moderno y su impacto en la educación intercultural". Sophia, colección de Filosofía de la Educación, No. 18 (2017). Recuperado el 24 de julio 2017. Disponible en 〈http://revistas.ups.edu.ec/index.php/sophia/article/ view/18.2015.12/134).

Yépez, Paula, Carolina Cedeño, Eduardo Granja y Tarquino Yacelga. “Aproximación a los enfoques de género e interseccionalidad: aprendizajes significativos en la interacción de estudiantes universitarios con una comunidad indígena del Ecuador". Revista Internacional de Ciencias Sociales, No. 6 (2017). 